\title{
Muons: civil applications
}

\section{Germano Bonomi*}

Department of Mechanical and Industrial Engineering - University of Brescia - via Branze 38, 25123 Brescia (Italy)

E-mail: germano.bonomieunibs.it

\begin{abstract}
Cosmic radiation has been known since the first decades of the 20th century: it has been considered for many years, the best source of projectiles to investigate the core of matter, from nuclei to elementary particles. Nowadays, cosmic ray muons are very important in particle and nuclear physics, because they are used for detector testing and calibration, and for detector alignment in complex measurement apparatuses, thanks to their high penetration capability. However, cosmic ray radiation has been already applied in fields beyond pure physics. An overview of the applications of the cosmic ray muons for civil usages, with special focus on the last development of the various techniques will be here presented.
\end{abstract}

53rd International Winter Meeting on Nuclear Physics, 26-30 January 2015

Bormio, Italy

\footnotetext{
*Speaker.
} 


\section{Introduction}

Primary cosmic rays come from the space, mostly originating from the sun. When entering the atmosphere they interact with the atoms of which it is composed and they generate showers that, according to the energy of the primary particle, can have an extremely high number of secondary particles. At the sea level about 10000 cosmic rays per minute and per square meter hit the ground. About 600 of them, in average, cross a human body every minute. The average energy is comprised between 3 and $4 \mathrm{GeV}$ and the flux is maximum at the zenith (vertical) and it scales approximately with $\cos (\theta)^{2}, \theta$ being the angle with respect the vertical. For more details about cosmic rays see [1] and references therein. When they hit the earth surface most of them are muons.

When they encounter the matter they 1) loose energy (by inelastic collisions, bremsstrahlungradiation, production of electron-positron couples, ... ) and 2) they deviate from the original trajectory (by multiple coulomb scattering) [2,3]. Basically the muons when crossing a given material they slow down, some time to rest being consequently absorbed, and are deflected from a straight line path. The weight of these effects depends on the type and thickness of the material that is being crossed and on the energy of the particle. Given a region of space to be inspected the overall muon incoming flux will be diminished when exiting the material and the angular distribution modified. Details of the volume crossed by the cosmic rays can be inferred by measuring such effects. The first applications were based on the "absorption/transmission" effect while in the last years new techniques based on the "scattering/deviation" effect have been proposed.

In the following (in Sec. 2 and Sec. 3 respectively) a list of both applications will be presented. Details of some of them, the ones in which our group have been involved and is actually working, as general examples, will be given. In particular, after a description of the basic principles of the muon tomography in Sec. 4, the Mu-Steel project will be described in Sec. 5. A new approach of the use of the cosmic rays for the stability monitoring of historical buildings will be finally presented in Sec. 6 .

\section{Applications based on the absorption effect}

The first ever civil application of the cosmic rays to inspect large volumes dates back to 1955 when the thickness of rock above a underground tunnel was measured by E. P. George [4]. This first application, as the ones that followed, was based on the measurement of the attenuation of the cosmic rays flux. By counting the number of muons entering and exiting the volume under inspection and assuming the composition of the crossed material, the total thickness of the material could be inferred. Another application much more spectacular was realized by the Physics Nobel Price L.W. Alvarez [5] that in 1970 made a "radiography" of the Chefren pyramid looking for hidden chambers ... finding none! See fig. 1 for a funny cartoon-description of the experiment. The same technique has been used to inspect the inner part of a vulcano [6,7]. This technique has been reutilized and improved more recently to visualize magma dynamics in an erupting volcano [8]. Other interesting and more curious applications have also been proposed and realized in the last years such as imaging of large vessels [9], cosmic muon detection for geophysical applications [10] and observation of the moon shadow [11]. 


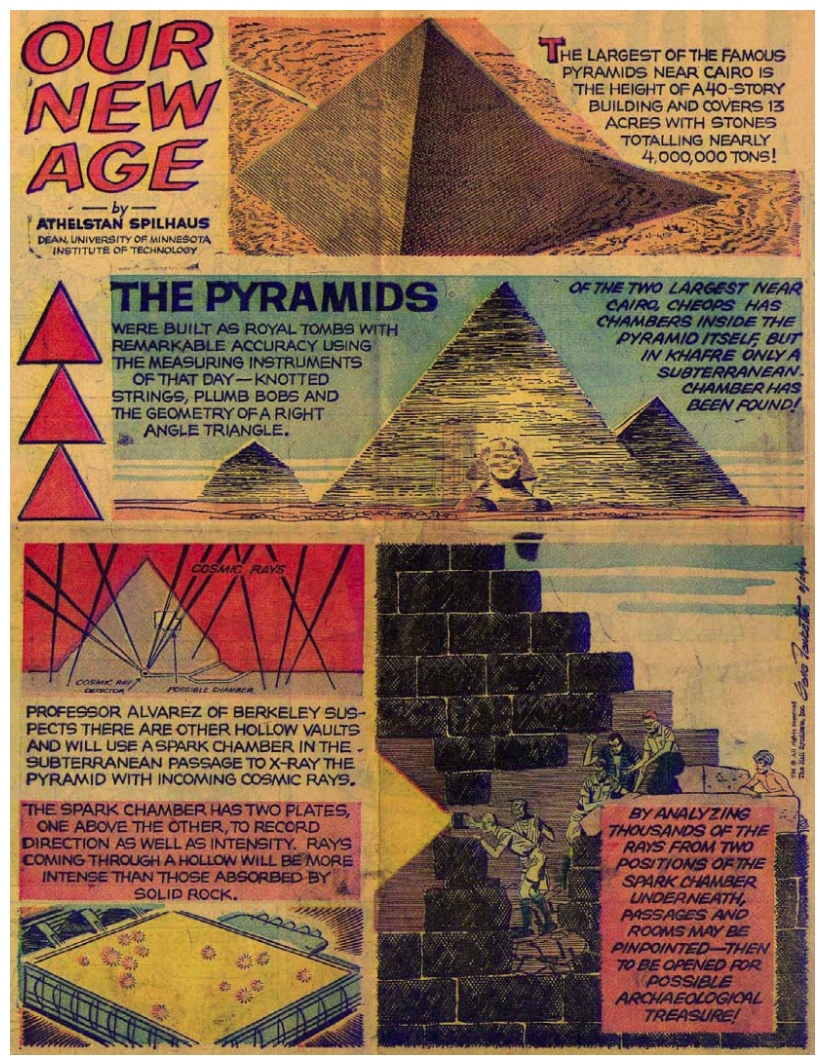

Figure 1: Funny cartoon-description of the Alvarez[5] experiment.

\section{Applications based on the scattering effect}

In 2002 a novel idea was proposed by a research group of the Los Alamos National Laboratory (LANL), to exploit the deflection of the cosmic rays to perform a tomography of a desired volume [12]. A working prototype of the dimensions of some tens of centimetres has been build and operated. It proved that in principle such technique can be used to scan large volumes. The basic idea is quite simple. To obtain the tomographic image, the multiple coulomb scattering [13, 14] of the muons with matter is exploited. Due to this interaction the direction of any charged particle travelling in matter is modified. The angular difference between the particle direction entering and exiting a given material depends on the thickness as well as on the nature of the material, in particular on a physical quantity called scattering density (see Sec. 4 for details), which is function of the examined material density and of its atomic number $Z$. The measurement of the angular deflection of a sample of cosmic muons allows, by means of a complex data analysis and image reconstruction software, to determine the distribution of the density inside the sample. In other words this technique can discriminate different materials inside the volume under inspection. Details of this technique, called muon tomography, will be reported in the following (see Sec. 4). Since the introduction of this new technique various applications have been proposed and realized. What follows is an extended list that doesn't pretend to be exhaustive, but it is useful to understand the variety of ideas and the numerousness of the research groups involved in this field. 
The Los Alamos group that originally proposed the technique indeed continued the study related to muon tomography and recently proposed to inspect the damaged cores of the Fukushima reactors [15]. This measurement was realized in 2015 and showed a complete meltdown of a Fukushima reactor. This study was performed in collaboration with a commercial spin-off from the LANL called Decision sciences[16]. The company was also awarded a contract with the U.S.A. Department of Homeland Security to build a large scale demonstrator of the muon tomography to inspect container at ports and borders. This portal was indeed constructed and its tests completed in April 2015. Another similar project is the Muon portal collaboration (see [17] and references therein) that plans to build a large area muon detector for a noninvasive inspection of shipping containers in the ports, searching for the presence of potential fissile $(\mathrm{U}, \mathrm{Pu})$ threats. The detector is based on scintillating-fibers. Also based on the same detecting technology, scintillating-fibers, is the Muon Tomography Project [18] of the University of Glasgow which goal is to build a system able to monitor nuclear waste containers. In Canada a similar system, called CRIPT - Cosmic Ray Imaging and Passive Tomography [19], is under construction. The project has been proposed to inspect cargo in shipping containers and to determine the presence of special nuclear materials. The University of Bristol on the other hand is trying to use a different type of detectors, the resistive plate chambers (RPC) to develop a system for the muon tomography [20]. To remain in the United Kingdom at the Boulby Underground Laboratory studies are underway to explore the use of muon tomography for deep 3D geological surveying applications [21]. A couple of European Commission projects were also financed $[22,23]$ and they took advantage of a large scale muon tomography demonstrator that was build at the Laboratori Nazionali di Legnaro of the INFN [24]. The first project (Mu-Steel) was dedicated to the inspection of container entering steel mills and took place between 2010 and 2012. The second project (Mu-Blast) is underway and is studying the possibility to inspect the inner part of a bust furnace with the cosmic rays. In 2007 a different approach, with respect to muon tomography cosmic ray was proposed [25] for measurement application in civil and industrial engineering, for the monitoring of alignment and stability of large civil and mechanical structures.

In the following, after a discussion of the basic principles of the muon tomography (Sec. 4), a detailed description of the Mu-Steel project, as a general representative case of a muon tomography application, will be given (Sec. 5), while an example of the new approach for the monitoring of the stability of historical building will be described separately (Sec. 6).

\section{Muon tomography: basic principles}

Muon tomography is based on multiple coulomb scattering of cosmic ray muons crossing the volume to be inspected. When a charged particle crosses a material, its trajectory is deflected from the direction of incidence. The distribution of the scattering angle $(\delta \theta)$ projected on a plane containing the incident particle trajectory is approximately Gaussian with zero mean value and variance related to the properties of the homogeneous crossed volume by the following approximate formula $[13,14]$ :

$$
\sigma^{2} \approx \frac{(13.6 \mathrm{MeV} / \mathrm{c})^{2}}{\beta^{2} \mathrm{p}^{2}} \frac{\mathrm{L}}{X_{0}}
$$


where $\mathrm{L}$ is the thickness of the crossed material, $X_{0}$ is its radiation length, $\mathrm{p}$ is the particle momentum and $\beta$ its velocity. Measuring the deflection of many particles it is therefore possible to infer the quantity

$$
\lambda=\frac{1}{\mathrm{X}_{0}}=\rho \cdot Z\left[\frac{(Z+1) \log 287 / \sqrt{Z}}{A \cdot 716.4 \mathrm{~g} / \mathrm{cm}^{2}}\right]
$$

which can be referred to as scattering density. As can be seen from (4.2), this quantity is strongly related to the mass density and atomic number of the crossed material. The projections of the scattering angle on two orthogonal planes are statistically independent.

An additional measured quantity is the displacement $(\delta x)$, defined as the difference of the impact point of the muon on the exit detector and the impact point expected in the absence of any scattering. For a given scattering angle, the displacement is proportional to the distance of the scattering object from the exit detector, therefore it gives information on the vertical position of the object. The use of the displacement improves the space resolution of the tomographic image.

Considering that the image reconstruction is still possible using only the information on the scattering angle, for the sake of simplicity and to avoid unnecessary complexity in the formalism, in the following description of the tomographic reconstruction procedure the use of $(\delta x)$ will be neglected.

Suppose now that a muon tomography of a large volume is performed placing two muon detectors above and below the inspection volume, as sketched in Fig. 2. We divide the full volume in volume elements, namely voxels, small enough to allow considering homogeneous the material distribution inside a single voxel. Let call $\lambda_{i}$ the unknown scattering density of the voxel $i$.

When a muon crosses the volume, muon $\mu_{1}$ in Fig. 2 for instance, the incoming and outgoing trajectories are measured. The true path is approximated with the broken line from entry point to the exit point through the Point of Closest Approach (PoCA). Within this approximation, the voxels traversed by the muon can be identified. The total scattering angle of the muon will be determined by the scattering density of all the voxels crossed by that muon, the ones marked in yellow. The trajectory of a different muon will be influenced by the densities of a different set of voxels, the green ones in Fig. 2 for muon $\mu_{2}$.

By the measurement of many muons crossing the inspected volume in different positions and at different angles, the scattering density of each individual voxel can be disentangled, as long as the individual voxel has been crossed by a sufficient number of muons.

More formally, the total variance of the scattering of the $i$-th muon through $\mathrm{N}$ voxels can be written as a function of the voxel scattering density and path length $\mathrm{L}_{i j}$ of the $i$-th muon inside the $j$-th voxel:

$$
\sigma_{i}^{2}=\frac{(13.6 \mathrm{MeV} / \mathrm{c})^{2}}{\mathrm{p}^{2}} \sum_{j=1}^{\mathrm{N}} \mathrm{L}_{\mathrm{ij}} \lambda_{j}
$$

Since in general the measurement of the muon momentum is impossible or very difficult, and the muon momentum spectrum is very broad, the previous equation is averaged over the muon momentum spectrum and the $p$ value is substituted by an "effective" momentum value $p_{\text {eff }}$. This approximation will lead to a degree of noise of the reconstructed image.

To build a useful 3D map, the contribution of the largest possible set of muons must be collected, covering different positions and trajectory angles in the reconstructed volume. From (4.3) 


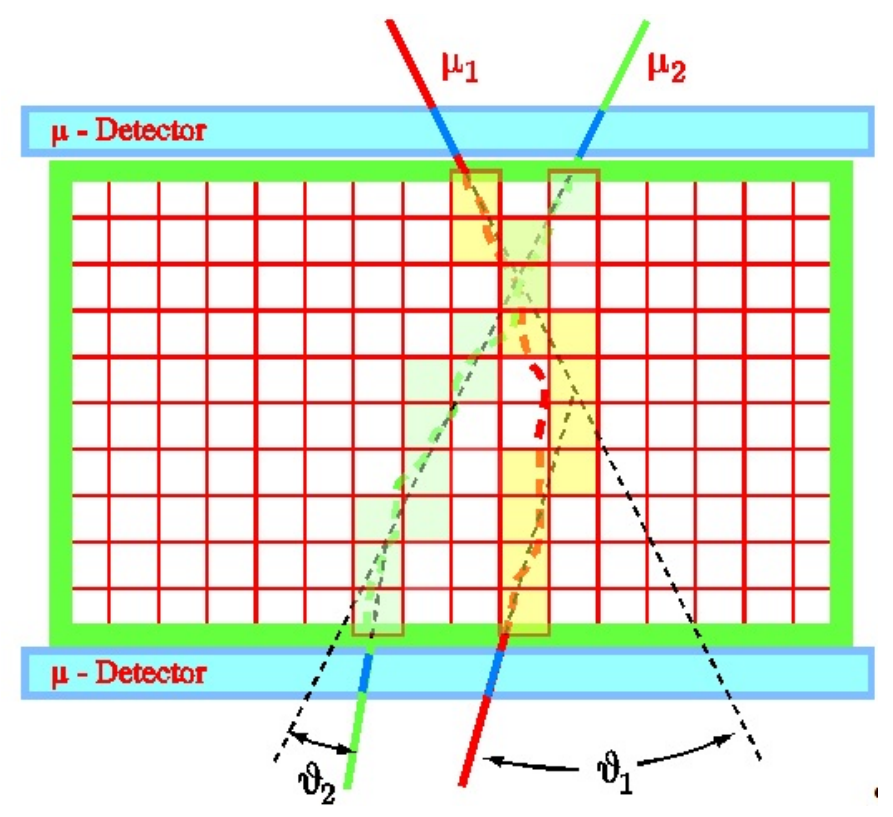

Figure 2: Hypothetic paths of two different muons inside the inspected volume. Muon $\mu_{1}$ is supposed to cross yellow voxels and muon $\mu_{2}$ the green ones.

with the fixed $p_{\text {eff }}$ value of the momentum, one can write the likelihood of the occurrence of the scattering angles measured in the sample of $\mathrm{M}$ muons as a function of the scattering density profile in the $\mathrm{N}$ voxels $\left(\underline{\lambda}=\lambda_{1}, \lambda_{2}, \ldots, \lambda_{\mathrm{N}}\right)$ :

$$
\mathrm{P}(\underline{\lambda})=\prod_{i=1}^{\mathrm{M}} \frac{1}{\sigma_{i}(\underline{\lambda}) \sqrt{2 \pi}} \exp \left(-\frac{\delta \theta_{i}^{2}}{2 \sigma_{i}^{2}(\underline{\lambda})}\right)
$$

Thus is possible to build the density profile $\underline{\lambda}$ in the volume by minimizing the logarithmic likelihood:

$$
\ln \mathrm{P}(\underline{\lambda})=\sum_{i=1}^{\mathrm{M}}\left[\frac{\delta \theta_{i}^{2}}{2 \sigma_{i}^{2}(\underline{\lambda})}+\ln \left(\sigma_{i}(\underline{\lambda})\right)\right]
$$

in the set of $\mathrm{N}$ variables $\underline{\lambda}$. This is achieved through an iterative Expectation-Maximization algorithm. This technique, proposed originally by [26, 27], is the base of the applications based on scattering listed in the previous section. In the following details of one of them, the Mu-Steel project, as a general example, will be given. 


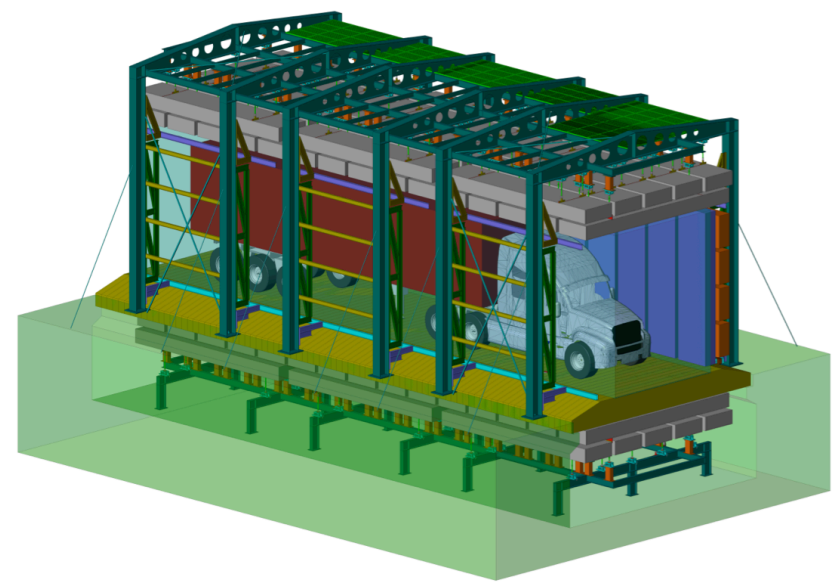

Figure 3: A schematic view of the Mu-Steel portal design. In order to guarantee easy access to the truck, the minimum safety zone to be kept clear along the length of the portal has been set to $5000 \mathrm{~mm}$ (width) $\mathrm{x}$ $4000 \mathrm{~mm}$ (height).

\section{Muon tomography: the Mu-Steel project as a detailed example}

One of the mayor concerns of the metal recycling industry is the accidental melting of radioactive sources[28]. Even though the reported events are rare (few events per year), the consequences can be extremely serious, with a huge environmental and economical impact on the workers, on the surroundings and on the industry. Indeed the melting can cause the radioactive contamination of workers, buildings, neighborhood, production lines, materials, etc.

The goal of the Mu-Steel project was to study the feasibility of building a portal based on muon tomography to monitor truck containers filled with scrap metals entering steel mills foundries to detect shielded radioactive sources. It is important here to underline that by means of the muon tomography, the portal is able to detect the shield of the source and not the source itself. For this reason, such a system would be used only in association with a traditional radiation portal. Specifically, the Mu-Steel project was aimed at (1) producing a complete design of a muon tomography portal, (2) building a detector prototype for the final portal and (3) developing the 3D tomographic software for a fast and efficient image reconstruction and radioactive source shield detection. Particular attention was devoted to the overall scanning and processing time in relation to the size of the shielding material to be detected along with the detection efficiency and the false positive percentage. A reasonable inspection time per truck had to fall in the range of 3 to 10 minutes. The mechanical design of the portal has been finalized with four muon detectors: upper detector, lower detector and two lateral detectors. A schematic view of the designed portal is shown in fig. 3 where a lateral detector has been removed in order to have an easy overview of the design.

The muon detector for the full portal has been designed as an assembly of units based on drift tubes technology. A detector unit prototype with $4000 \mathrm{~mm}$ long tubes has been built and operated. It met the requirements of the project, delivering an angular resolution of a few mrad, a hit resolution of a few hundred microns, and an overall efficiency close to $90 \%$.

As previously stated, the ultimate goal of the project was to demonstrate the capability of the 
system to detect a small radioactive source shielded inside a $75.6 \mathrm{~m}^{3}$ truck container volume filled with metal scraps in an average acquisition time of few minutes. The image reconstruction starts from the muon path tracking measuring, with the muon detectors placed above and below the truck, the trajectories for both the entering and the exiting paths. The analyzed volume lies inside the entering and exiting detectors. The simplest method that can be used is the so called Single Scattering Approximation. In space this means utilizing the Point of Closest Approach (PoCA) of two straight lines. It surely works when the data are characterized by a single scattering point (i.e., there is a single heavy element box inside an empty container), but it fails when several scattering centers are present, indeed it doesn't use all the information in the sample of muons. A more efficient approach is to divide the volume into $\mathrm{N}$ cubic voxels in which the scattering density is assumed to be constant, as described in details in Sec. 4. The volume was thus divided into 604800 voxels, each with a volume of $5 \times 5 \times 5 \mathrm{~cm}^{3}$, to allow the detection of a 2 or 5 later radioactive source shield, corresponding to a $13 \mathrm{~cm}$ and $17 \mathrm{~cm}$ side cube respectively in a time interval of 3-10 minutes. To meet the goal, the overall computing time was optimized using parallel processing, cache alignment, memory optimization, application specific-libraries, and other techniques. To test the overall reconstruction software, the algorithms were tested with a specific complete GEANT4 [29] simulation that included a muon generator engine, a model of the full portal and of the truck container filled with scrap metal of average density of $0.5-0.7 \mathrm{~g} / \mathrm{cm}^{3}$, obtained with cubes of random density ranging from 0 to $7.87 \mathrm{~g} / \mathrm{cm}^{3}$, the density of iron. The GEANT4 simulation was tuned to match the experimental results obtained with real data of the INFN-LNL muon tomography prototype described in [24]. In particular, a lead block was hidden in a small container filled with scrap metal and positioned in the muon tomography prototype. Both real data and simulated ones were compared to tune the Monte Carlo that could then be used to simulate the Mu-Steel full portal. The 3D reconstructed images were then post-processed with specific filters to improve the density contrast. In particular, various filters were tested, namely the median, the Gaussian and a combination of $\alpha$-trimmed and Gaussian filters, the latter being the most effective one. The effect of the filtering process is displayed in fig. 4 (upper) that shows the tomographic 3D density image reconstruction of a truck container filled with scrap metal, in which a 5 liter source shielding (lead box) was hidden. The image corresponds to a data taking time equivalent to 5 minutes of real cosmic rays. Even if the battery, some details of the gear box, and more importantly, the lead shielding are visible in the reconstruction, the use of the filters was not sufficient for an efficient source shield detection algorithm.

A notable limit in using the scattering information of each muon for the image reconstruction is that the momentum is unknown. Indeed, large scattering can be obtained with both a low momentum muon crossing low density material or with a high momentum muon crossing high density material. To overcome such a limit and to further improve the image reconstruction, a muon momentum estimate was developed and used. In particular, the scattering of the muons in the detector itself, when crossing the aluminum drift tube walls was exploited. The deviation from a straight line, in the detector itself, is indeed correlated to the muon momentum (the bigger the momentum, the smaller the deviation). A rough but very valuable muon momentum estimate could thus be derived. The use of such information proved to greatly refine the image reconstruction as can be seen in fig. 4 (lower), where the effect of using the momentum estimate is clearly visible.

The reconstructed density image shown in fig. 4 (lower) demonstrates qualitatively that the 


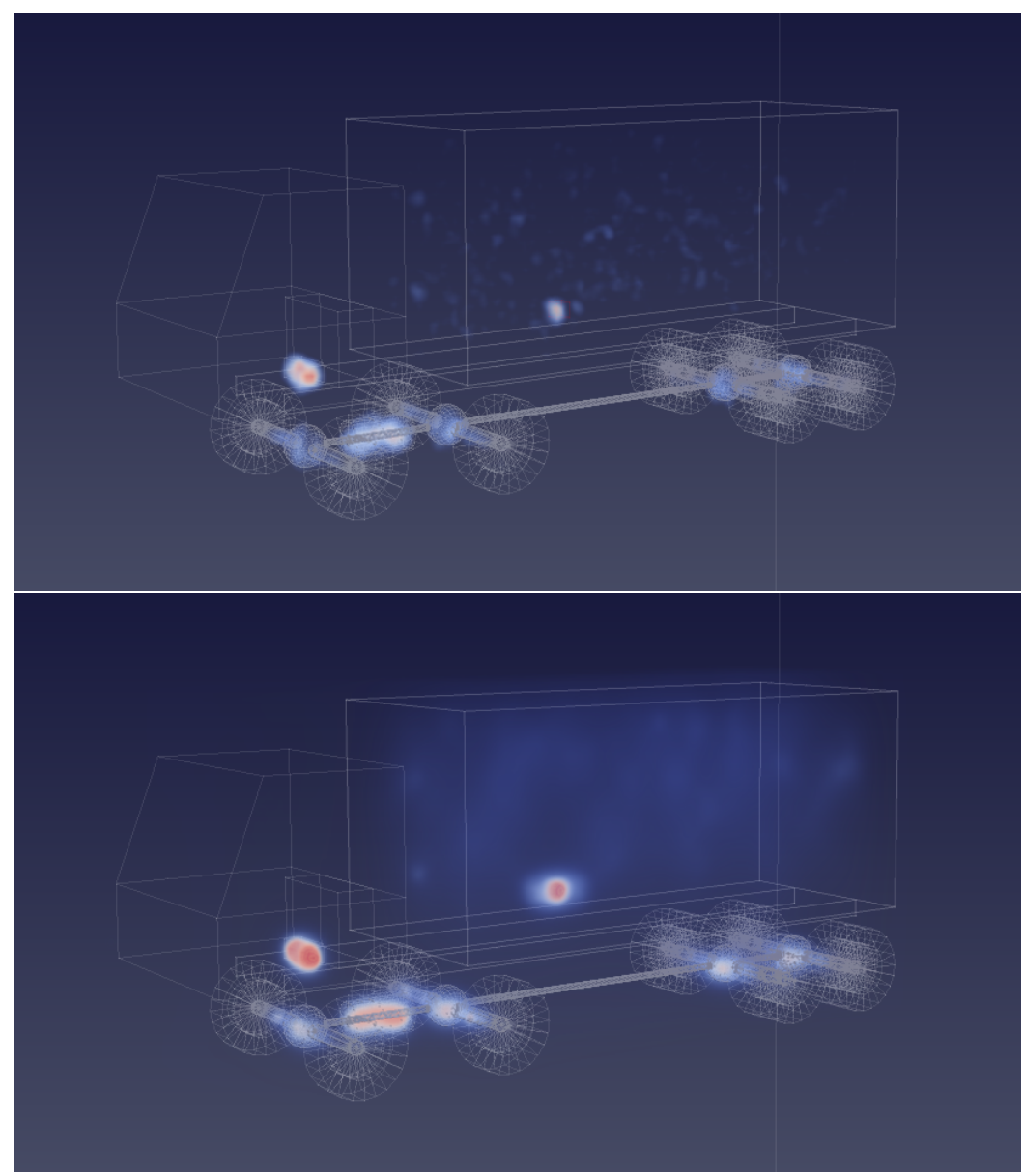

Figure 4: Tomographic 3D density image reconstruction of a Monte Carlo sample corresponding to 5 minutes of real cosmic data taking for a scrap metal container hiding a 5 liter source lead shielding: (upper) applying a combination of $\alpha$-trimmed and Gaussian filters and then (lower) adding the muon momentum estimate.

system is able to detect a 5 liter radioactive source shield in 5 minutes. To quantify such a capability, we introduce two important variables: (1) the undetected source shield fraction ("How many trucks with a source shield WILL NOT trigger an alarm?") and (2) the false alarm fraction ("How many trucks without a source shield WILL trigger an alarm?"). Clearly, to state that a source shield is present, a detection threshold on the voxel density has to be set. A "too low" threshold would imply a good efficiency but a high false alarm ratio, while a "too high" threshold would mean a low false alarm ratio, but a low efficiency. A perfect system would have both a zero undetected ratio and a zero false alarm ratio.

The results are summarized in two curves that show, as a function of the detection threshold, the ratio between the number of undetected source shields and the total number of samples in which the source shield was present (so called FNF, False Negative Fraction, curve) and the false alarm ratio, that is the ratio between the number of "detected" source shields and the total number of samples in which the source shield was absent (so called FPF, False Positive Fraction, curve). In 


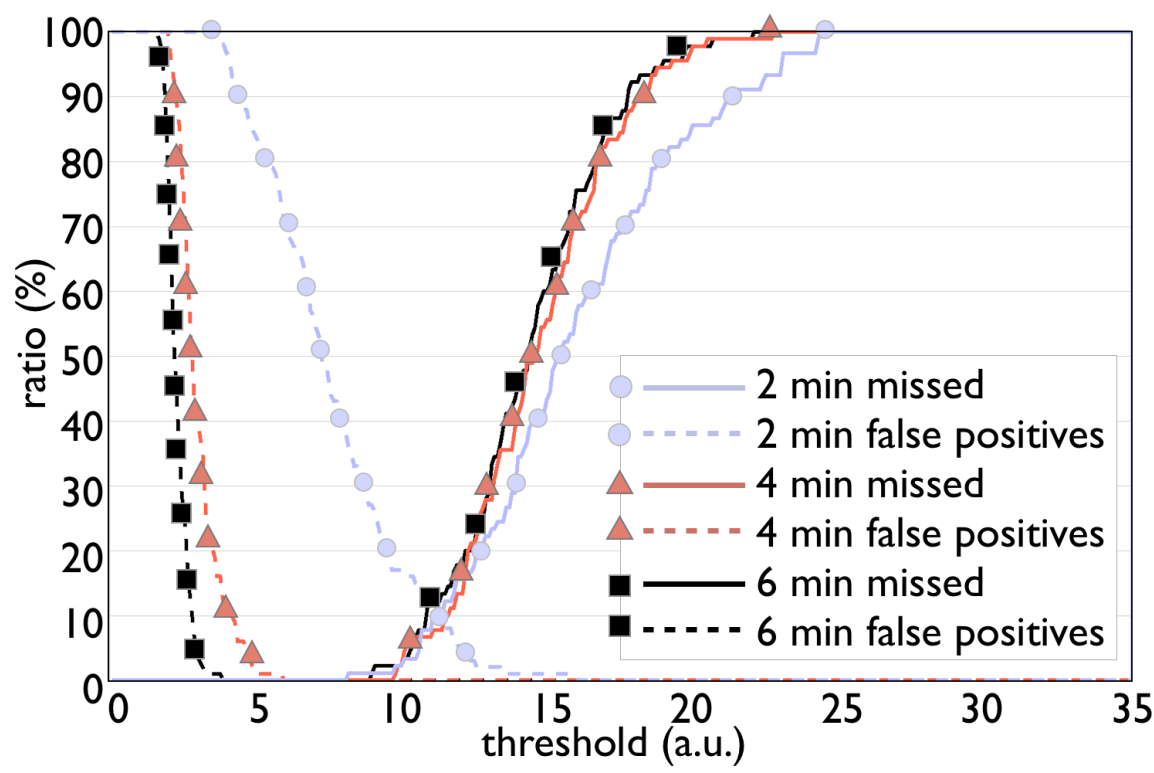

Figure 5: The FPF/FNF curves for a 5 liter source shielding.

fig. 5, the FPF/FNF curves for a 5 liter source shield and for 3 different data acquisition times are shown. In 2 minutes, the system can perform a $10 \%$ false alarms ratio with a detection efficiency of about $90 \%$. On the other hand, in 4 minutes, there is a threshold range in which there are $0 \%$ false alarms and 100\% detection efficiency. The same result can be obtained in 6 minutes for a 2 liter source shielding. The obtained results proved that muon tomography is a suitable technology for detecting shielded radioactive sources hidden in scrap metal containers.

\section{Stability monitoring by cosmic rays}

As previously stated in nuclear and particle physics, muons are often used to "calibrate" the experimental apparatuses, that is to measure the relative position of different detectors with respect one to each other. Few years ago our research group proposed to use a similar technique for civil applications such as the mechanical monitoring of an industrial press [25]. Since muons are like (almost) straight lines and they can easily cross floors and walls of buildings, a new application for the stability monitoring of historical buildings has also recently been proposed and studied [30, 31]. The main component of the suggested monitoring system is a muon-telescope, shown schematically in fig. 6(a). It is composed by a set of three muon detector modules supported by an appropriate mechanical structure and axially aligned at distance of $50 \mathrm{~cm}$ one from the other. Each module is composed by two orthogonal layers of 120 scintillating optical fibers with $3 \mathrm{~mm} \times 3 \mathrm{~mm}$ cross section and $400 \mathrm{~mm}$ length, as shown in fig. 6(b).

The two planes of orthogonal scintillating fibers provide the measurement of the crossing position of an incident muon in the $\mathrm{x}$ and $\mathrm{y}$ coordinates, with a pitch of $3 \mathrm{~mm}$. Considering a flat detection efficiency over the entire surface of the scintillating fiber, the expected spatial resolution on the hit coordinate is about $0.9 \mathrm{~mm}$. 

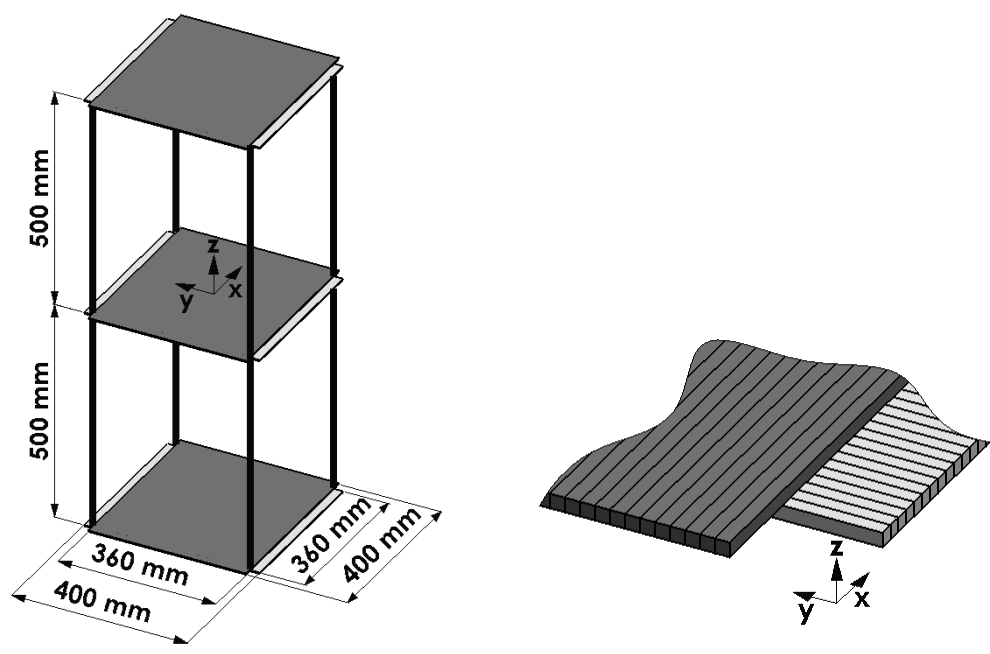

Figure 6: (a) Structure of the muon-telescope formed by three muon detector modules axially aligned at a distance of $50 \mathrm{~cm}$ each other. (b) Sensitive volume of the muon detector module formed by two orthogonal layers of 120 scintillating fibers $3 \mathrm{~mm} \times 3 \mathrm{~mm}$ cross section and $400 \mathrm{~mm}$ length.

The muon-telescope is mechanically fixed to a structural element of the building, that constitutes the reference system, with its axis aligned in the direction corresponding to the part of the structure whose displacements should be monitored. A fourth muon detector module, with the same geometry and structure of the previous ones, is positioned as muon-target on the point to be monitored.

Thanks to their high penetrability, cosmic ray muons are able to cross the system of four detectors as well as the interposed building structures. In this way it is possible to continuously monitor the horizontal displacements of the muon-target relative to the muon-telescope fixed on the masonry structure of the building.

Indeed, the trajectory of a cosmic ray muon crossing the system of four detectors can be extrapolated from the muon-telescope to the plane of the muon-target detector, in the hypothesis that it is a perfect straight line. The difference between the muon crossing point on the muon-target and the extrapolated one from the muon-telescope allows the position of the muon-target relative to the muon-telescope to be measured. Possible displacements of the position of the muon-target relative to a reference position previously determined can be inferred.

In crossing the interposed materials, the trajectories of cosmic ray muons suffer multiple scattering angular deviations. At fixed momentum and at low deviation angles, these deviations follow a Gaussian law with variance depending on the inverse square of the muon momentum $[13,14]$. Being these stochastic effects largely dominant over intrinsic detector resolution and geometrical conditions, statistical distributions of the difference between measured crossing coordinates in the muon-target and the predicted crossing coordinates determined by extrapolation from the muontelescope are therefore necessary, in order to reduce the stochastic effects by statistical inference methods. As shown in [25], efficient unbiased estimators of the systematic displacement can be 
extracted from these distributions. To test the validity of the proposed method a Monte Carlo simulation based on GEANT4 has been performed for a specific case, that is the static anomalies of the wooden vaulted roof of the "Palazzo della Loggia" of the City of Brescia.

\subsection{Application to a specific case: "Palazzo della Loggia"}

Since its completion in 1574, the "Palazzo della Loggia", seat of the municipal hall in the town of Brescia, has cumulated a long sequence of injuries, transformations, repairing interventions, some of which have generated considerable problems of structural stability of the building. The grandiose wooden vaulted roof was completely reconstructed in 1914, with the same architectural shape and construction techniques of the original one, destroyed by a fire one year after the completion of the building. The shape of the dome is like an upside down ship which reaches in elevation a maximum of $16 \mathrm{~m}$, having the planar rectangular sides of about 25 and $50 \mathrm{~m}$ respectively. The structural architecture of the vault consists of principal truss wooden arches and simple secondary arches, both connected at the top by a truss made wooden beam.

Immediately after its construction, the present wooden vaulted roof structure exhibited a progressive deformation of the longitudinal top beam and of the key points of the connected arches. The progressive deflection of the top beam was measured to be $190 \mathrm{~mm}$ in 1923, $520 \mathrm{~mm}$ in 1945 , $800 \mathrm{~mm}$ in 1980. Starting from 1990, a systematic campaign of investigation and monitoring of the different stability problems of the Palace (in the following "the measurement campaign") has been committed by the Brescia municipality to the "Centro di studio e ricerca per la conservazione e il recupero dei beni architettonici e ambientali dell'Università di Brescia” [32, 33]. In particular, the progressive deformations of the principal arches of the wooden vault have been studied with a specifically designed mechanical measurement system. A progressive collapse of the wooden structure of the arch of about $1 \mathrm{~mm}$ per year has been measured.

\subsection{Simulations and results}

The features and expected performances of the proposed measurement system were studied by Monte Carlo simulations using the GEANT4 package. A cosmic ray muon generator based on experimental data was implemented in the code in order to simulate as realistically as possible the momentum, the angular distribution and the charge composition of the cosmic ray radiation at the sea level [34].

In order to study the performances of the proposed monitoring system, the structure and composing materials of the muon-telescope and muon-target were modeled as well as the relevant structures of the "Palazzo della Loggia" building.

Three configurations were considered: the first with the muon-target located $0.50 \mathrm{~m}$ above the wooden ceiling of the "Salone Vanvitelliano" (this position will be pointed as "P1" in the following), at the first floor of the Palace; the second with the muon-target located $5.8 \mathrm{~m}$ above the wooden ceiling ("P2"); the third with the muon-target located $10.0 \mathrm{~m}$ above the wooden ceiling ("P3"). In the three different conditions the muon-telescope was located on the vertical of the corresponding muon-target, $3.0 \mathrm{~m}$ below the wooden ceiling. The ceiling of the large "Salone Vanvitelliano" was modeled as a bulky $15.0 \mathrm{~cm}$ thick wooden layer. 

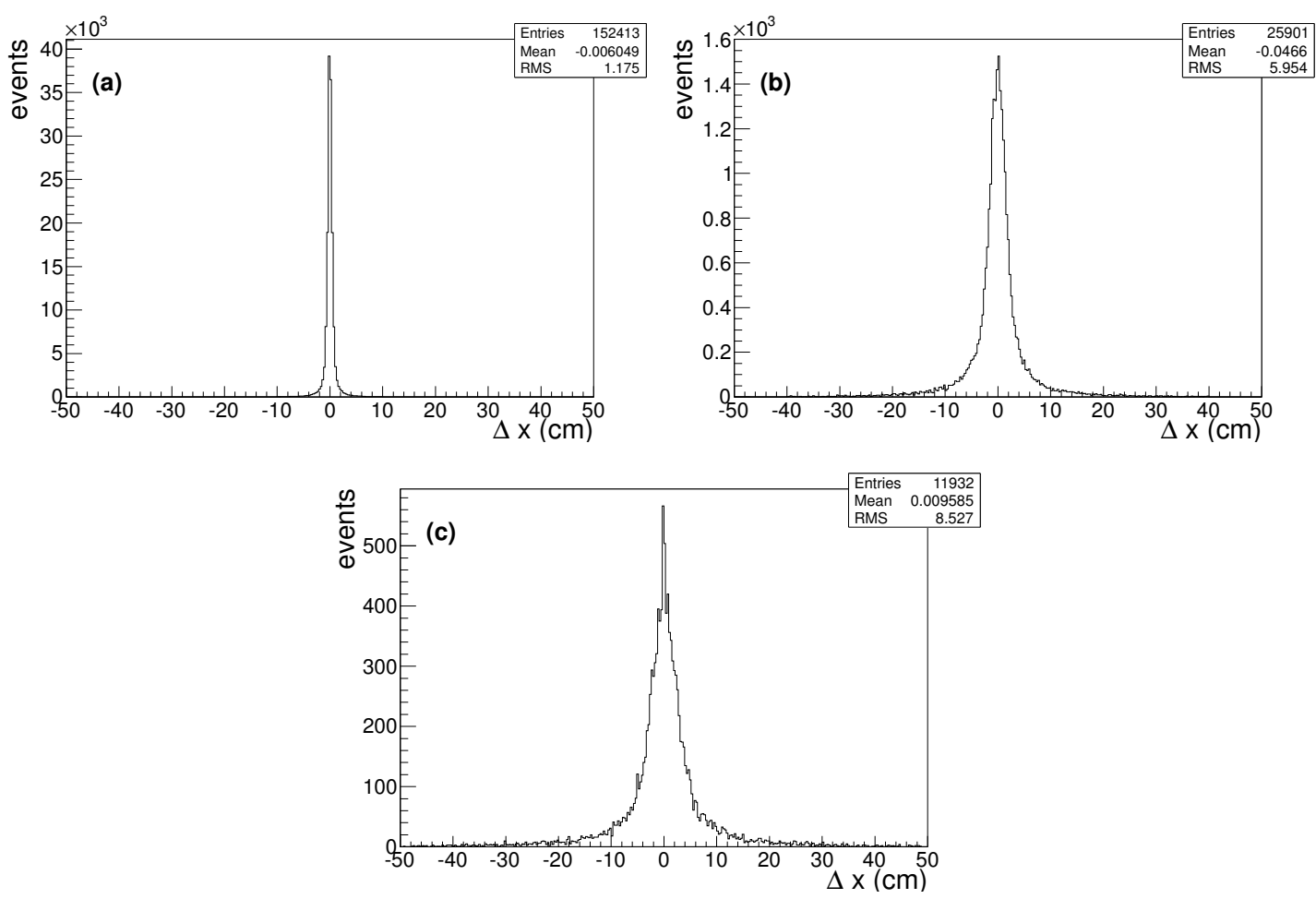

Figure 7: Distributions of the differences $\Delta x$ between the crossing point coordinates measured on the muontarget in position P1 (a), P2 (b) and P3 (c), and the crossing point coordinates of the extrapolated muon trajectory measured by the muon-telescope, in 15 days data taking time.

\subsection{Position measurement uncertainty of the stability monitoring system versus data taking time}

Simulation campaigns of populations of cosmic ray muons crossing the measurement system were performed for the three configurations described above. The distributions of the differences $\Delta x$ and $\Delta y$ between the crossing point coordinates measured by the muon-target and the crossing point coordinates extrapolated from the muon-telescope were calculated.

In figs. 7, sample distributions $\Delta x$ for the three configurations are shown, for an elapsed data taking time of 15 days, corresponding to about $31.7 \times 10^{6}$ cosmic ray muons crossing the muontarget surface at the rate of 170 muons $/\left(\mathrm{s} \mathrm{m}^{2}\right)$ [1]. The $\Delta y$ distributions are not shown, since they are statistically identical to the $\Delta x$ distributions.

As the muon-target and the muon-telescope are exactly coaxial in the simulation, the $\Delta x$ distributions are symmetric and centered at zero. The shape of the distributions exhibits a central narrow peak with very long tails on both sides. This shape is due both to the intrinsic uncertainty of the muon-telescope in measuring the direction of the cosmic ray muon and to multiple scattering angular deviations of the muon trajectories traversing the interposed materials. The latter effect dominates for large distances of the muon-target from the muon-telescope.

The long tails of the distributions are due in part to low momentum muons, suffering larger deviations, and, in part, to spurious events corresponding to emission of delta rays, most of which 


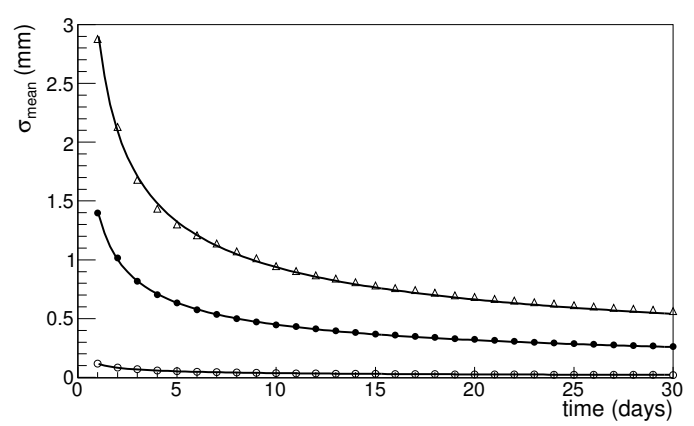

Figure 8: Relation between the standard uncertainty on the mean value of the sample distribution and the data taking time, for the muon-target in position P1 (०), P2 $(\bullet)$ and P3 $(\triangle)$.

can be discarded with a more refined data analysis. At present, the only selection applied to these bad quality events is an arbitrary cut of both tails in the three distributions, discarding about $1 \%$ of the total events.

The mean value of the sample distributions represents an unbiased estimator of the position of the muon-target relative to the muon-telescope axis. The root mean square of the sample distribution represents the uncertainty in the measurement of the position of the muon-target relative to the muon-telescope.

The uncertainty on the mean value is given by the well known relation $\sigma_{\text {mean }}=\sigma_{d i s t r} / \sqrt{N_{e v}}$ where $N_{e v}$ is the number of events in the distribution. Since in the same geometrical conditions the number of events in the sample distribution is proportional to the data taking time, the measurement standard uncertainty depends only on the inverse of the square root of the data taking time. In fig. 8 the relation of the position measurement standard uncertainty and the data taking time for the three examined conditions is plotted up to a data taking time of one month.

As time increases, the measurement standard uncertainty decreases. By fitting the plots with the general relation: $\sigma_{\text {mean }}=C / \sqrt{t}$ where $C$ is a constant depending on the geometry and materials interposed and $t$ is the data taking time expressed in days, the following values for the constant $C$ are obtained in the three conditions considered: $0.12 \mathrm{~mm}_{\text {day }}{ }^{1 / 2}, 1.42 \mathrm{~mm}_{\text {day }}{ }^{1 / 2}, 2.96 \mathrm{~mm} \mathrm{day}^{1 / 2}$; the errors from the fit procedure are below $1 \%$.

For example, in one month of data taking in position P3, where muon-target and muontelescope are positioned $13.0 \mathrm{~m}$ far apart, a measurement standard uncertainty of the order of $0.5 \mathrm{~mm}$ may be achieved. The same standard uncertainty may be achieved in a week of data taking in position P2, whereas a $0.1 \mathrm{~mm}$ may be measured in just one day in the position P1.

As expected, the standard uncertainty of the measurement system depends on the geometrical configuration considered, since both the root mean square of the distributions and the rate of useful events collected are strongly dependent on the geometry of the system and on the amount of materials interposed. Nevertheless, although requesting different data taking times, the position monitoring of all the three inspected points by a cosmic ray tracking system could provide performances compatible with the requested precisions and with the time scale characteristic of the inspected deformation phenomenon. Typical time scales, in the case of "Palazzo della Loggia" and, 

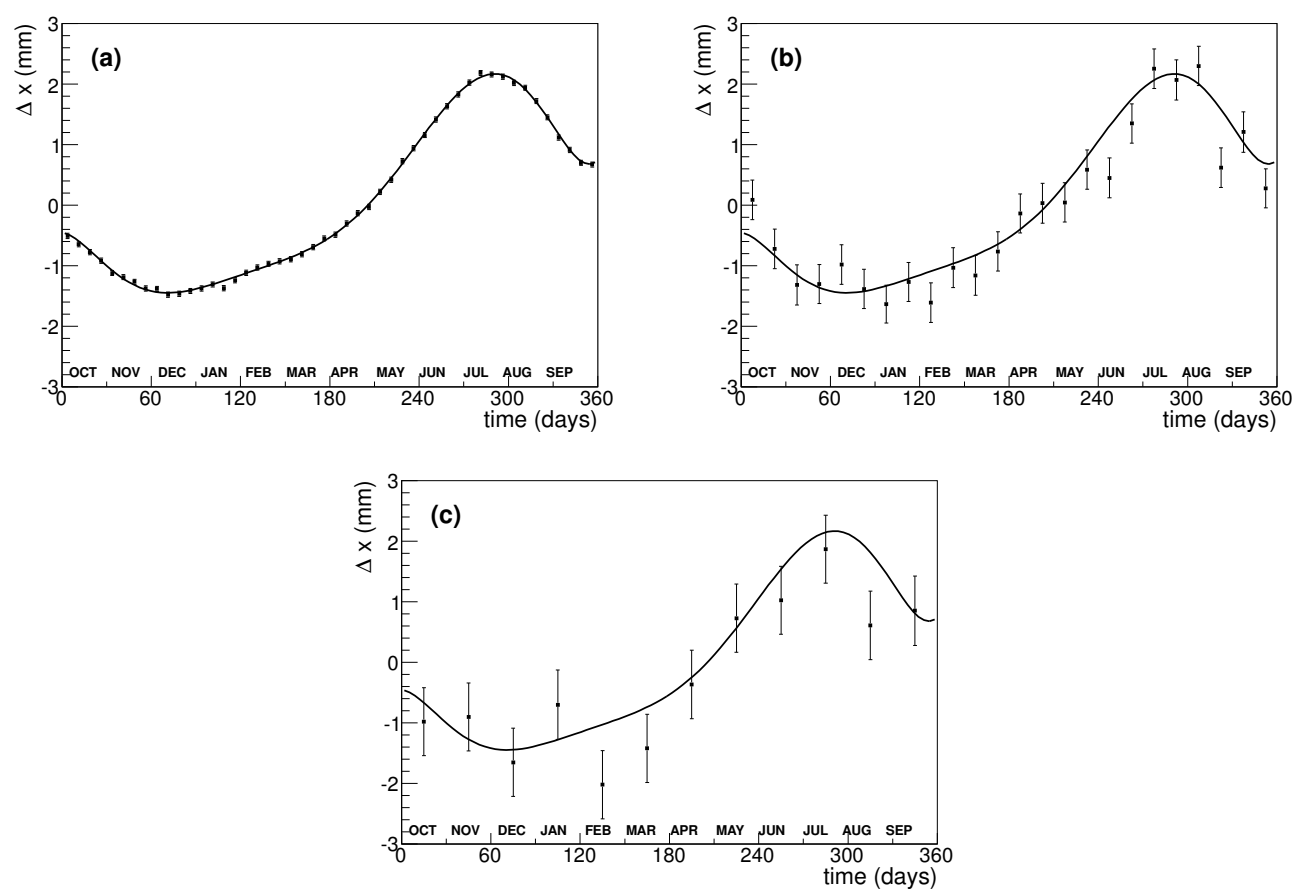

Figure 9: The seasonal deformation evaluated by the measurement campaign [20], with superimposed the result of the simulation measurements with the sample mean of the position of the muon-target displaced following the assumed structure deformation, with sampling rate of one week for position P1 (a), two weeks for position P2 (b) and one month for position P3 (c).

in general, for historical buildings, may span over several years. Furthermore, as demonstrated in this case and unlike other monitoring systems, a stability monitoring system based on tracking of cosmic ray muons can efficiently operate also when the building points to be monitored are not reciprocally visible and are separated by solid masonry structures.

Due to the cosmic rays rate, a monitoring system based on muons tracking can't provide high precision results in short time. Therefore, it can be competitive with other monitoring techniques only when the deformation under study develops over periods of months or years and the requirements for the monitoring system is to track the slow deformation with time.

This is the case of the cyclic seasonal deformations of the wooden vaulted roof of the "Palazzo della Loggia", which have been simulated with the Monte Carlo program for points P1, P2 and P3 of the roof structure. As a realistic model of the seasonal deformation, the measured displacement in point $\mathrm{P} 2$ on the arch reins, reported by the measurement campaign [32, 33], was adopted.

In fig. 9 the curve corresponding to the assumed seasonal deformation (the same for the three points) is shown as a continuous line. The results of the simulated measurements of the position of the muon-target, displaced following the assumed structure deformation, are shown; sampling rates of one week, two weeks and one month respectively for points P1, P2 and P3 have been used. It is evident the ability of the proposed measurement system to follow seasonal structural displacements of few millimeters and, consequently, also systematic ones.

A small prototype, consisting of three planes of scintillating fibers, has been built and it is 
being operated to show the capabilities of such a system to detect the relative displacement of one of three planes with respect to the other two. Results of the corresponding data taking will be subject of a dedicated publication.

\section{Conclusions}

Cosmic rays have been used in particle and nuclear physics for decades for detector calibrations and alignments. In the last years these standard techniques in research have been proposed for novel civil applications. There is now a multitude of examples of different approaches around the world, most of them are in the stage of feasibility studies while some of them already proved their reliability. All these applications exploit the fact that cosmic rays are a natural, safe and ubiquitous source of radiation since muons are available always and everywhere. The main efforts are now dedicated to the construction of prototypes and demonstrators and to the choice of the best detector featuring the characteristics suitable to satisfy the requirements of each specific system. It is reasonable to foresee that in a short time at least some of them will pass the research stage and will make their way to civil applications through industrialization and commercialization.

\section{References}

[1] J. Beringer et al. (Particle Data Group), Phys. Rev. D86, 010001 (2012)

[2] W. R. Leo, "Techniques for nuclear and particle physics experiments" (Springer-Verlag, Berlin 1987)

[3] G.F. Knoll, Radiation detection and measurements (Wiley, New York 1989)

[4] E.P. George, Commonwealth Engineer, July 1, 1955, p. 455.

[5] L.W. Alvarez et al., Science 167 (1970) 832.?

[6] K. Nagamine et al., Nucl. Instr. and Meth. A 356 (1995) 585.

[7] H. Tanaka et al., Nucl. Instr. and Meth. A 507 (2003) 657.

[8] H. Tanaka et al., Nature Communications 5 (2014), doi:10.1038/ncomms4381.

[9] P. M. Jenneson et al., Chemical Engineering Journal 130 (2007) 75.

[10] L. Oláh et al., Advances in High Energy Physics (2013) 560192 (doi 10.1155/2013/560192)

[11] Yu. M. Andreyev et al., Cosmic Research 40 (2002) 604 - A. V. Karelin et al., Cosmic Research 53 (2015) 128.

[12] K.R. Borozdin et al., Nature 422 (2003) 277.

[13] G.Z. Moliere, Z. Naturforsch. 2a (1947) 133; G.Z. Moliere, Z. Naturforsch. 3a (1948) 78.

[14] H.A. Bethe, Phys. Rev. 89 (1953) 1256.

[15] K. R. Borozdin et al., Physical Review Letters, 109 (2012) 152501

[16] http://www.decisionsciencescorp.com.

[17] P. La Rocca et al. JINST 9 (2014), C01056.

[18] R. Kaiser et al., EPJ Web of Conferences 66 (2014) 10005, doi:10.1051/epjconf/20146610005 
[19] J. Armitage et al., Int. Jou. Mod. Phys. Conf. Ser., 27 (2014) 1460129

[20] P. Baesso et al. JINST 9 (2014 ) C10041, doi:10.1088/1748-0221/9/10/C10041

[21] http://www.stfc.ac.uk/Boulby/

[22] G. Bonomi et al., Int. Jou. Mod. Phys. Conf. Ser., 27 (2014) 1460157 [European Commission Mu-Steel project (RFCS - CT-2010-000033)]

[23] European Commission Mu-Blast project (RFCS-630643) [2014-2016].

[24] S. Pesente et al., Nuclear Instruments and Methods in Physics Research A 604 (2009) 738.

[25] I. Bodini, G. Bonomi, D. Cambiaghi, A. Magalini and A. Zenoni, Meas. Sci. Technol. 18 (2007) 3537.

[26] L. J. Schultz et al., Nucl. Instrum. Meth. A, 519 (2004) 687

[27] L. J. Schultz et al., IEEE Transaction Image Process., 16 (2007) 1985

[28] D. S. Harvey, K. Baldry and A. Bishop, La Revue de Métallurgie-CIT, 285 (April 2004).

[29] S. Agostinelli et al., Nucl. Instr. Meth. A 506, 250 (2003); J. Allison et al., IEEE Trans. Nucl. Science 53, 270 (2006).

[30] A. Donzella, Il nuovo cimento, Vol. 37 C (2014) 223

[31] G. Bonomi et al., Proceedings of the 28th European Conference on Modelling and Simulation, ISBN: 978-0-9564944-8-1

[32] A. Franchi, E. Giuriani, A. Gubana, G. Lupo, G. Mezzanotte, P. Ronca and V. Volta, Per la conservazione del Palazzo della Loggia di Brescia - Parere sulla stabilità strutturale Centro di studio e ricerca per la conservazione e il recupero dei beni architettonici e ambientali, Dipartimento di Ingegneria Civile, Università di Brescia (Grafo edizioni, Brescia) 1993.

[33] A. Bellini el at. Il Palazzo della Loggia di Brescia - Indagini e progetti per la conservazione, Atti del Convegno di studi: "Storia e problemi statici del Palazzo della Loggia di Brescia", Università degli Studi di Brescia - Facoltà di Ingegneria, ottobre 2000 (Starrylink editrice, Brescia) 2000.

[34] L. Bonechi, Proceedings of the $29^{\text {th }}$ International Cosmic Ray Conference, (Pune) (2005) 101. 